\title{
Implementation of a Parameterized Interacting Multiple Model Filter on an FPGA for Satellite Communications
}

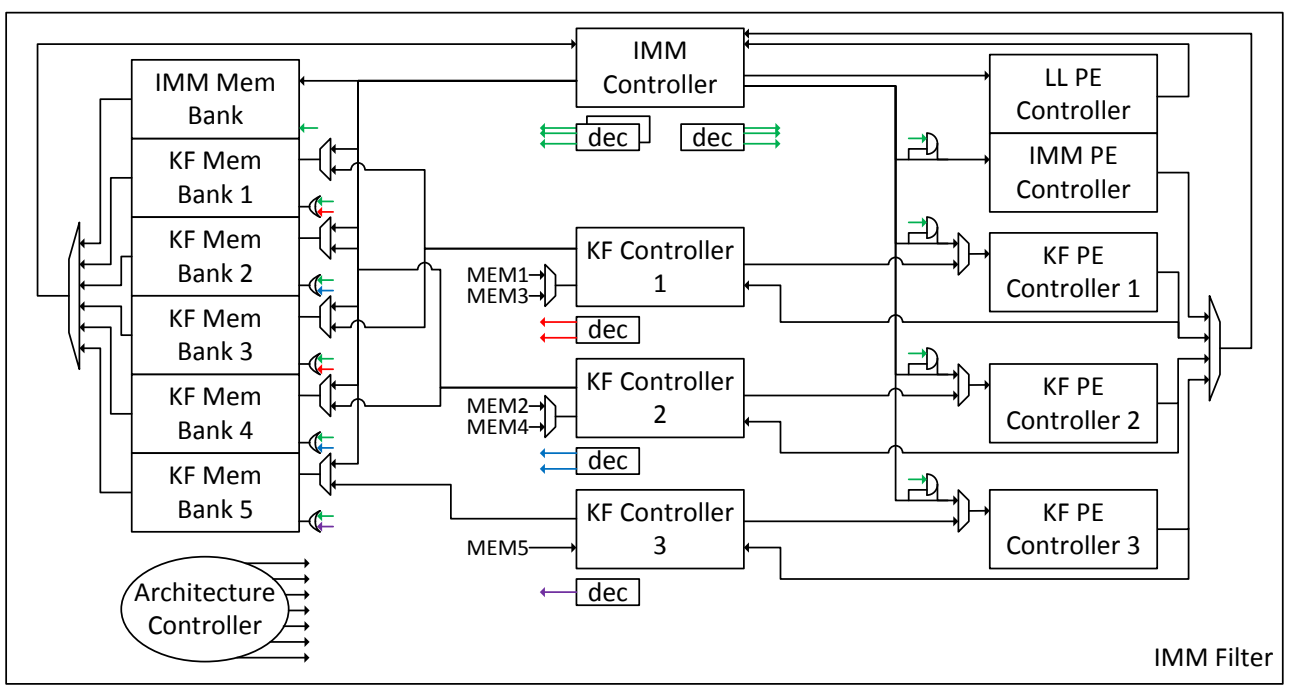

\author{
Timothy M. Hackett \\ Sven G. Bilén \\ Paulo Victor R. Ferreira \\ Alexander M. Wyglinski \\ Richard C. Reinhart
} 16 November 2016 number NNX15AQ41H) and a cooperative agreement with NASA John H. Glenn Research Center (grant number NNC14AA01A). 


\section{Overview of Research}

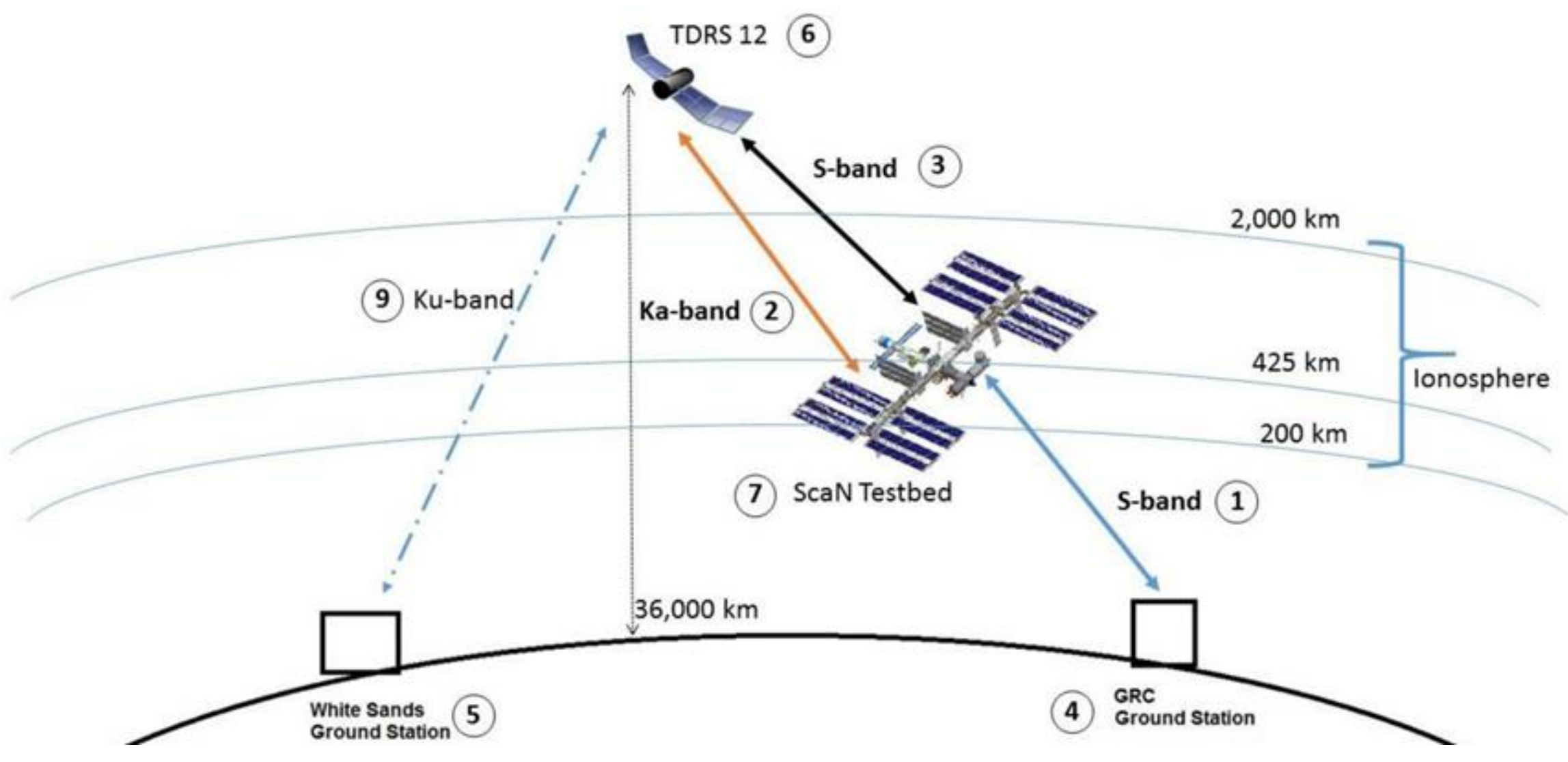




\section{Targeted Platform}

\section{JPL Radio}

- 66-MHz SPARC processor

- 2 x Xilinx Virtex-II FPGAs

- S-band transceiver

- L-Band (GPS) receiver

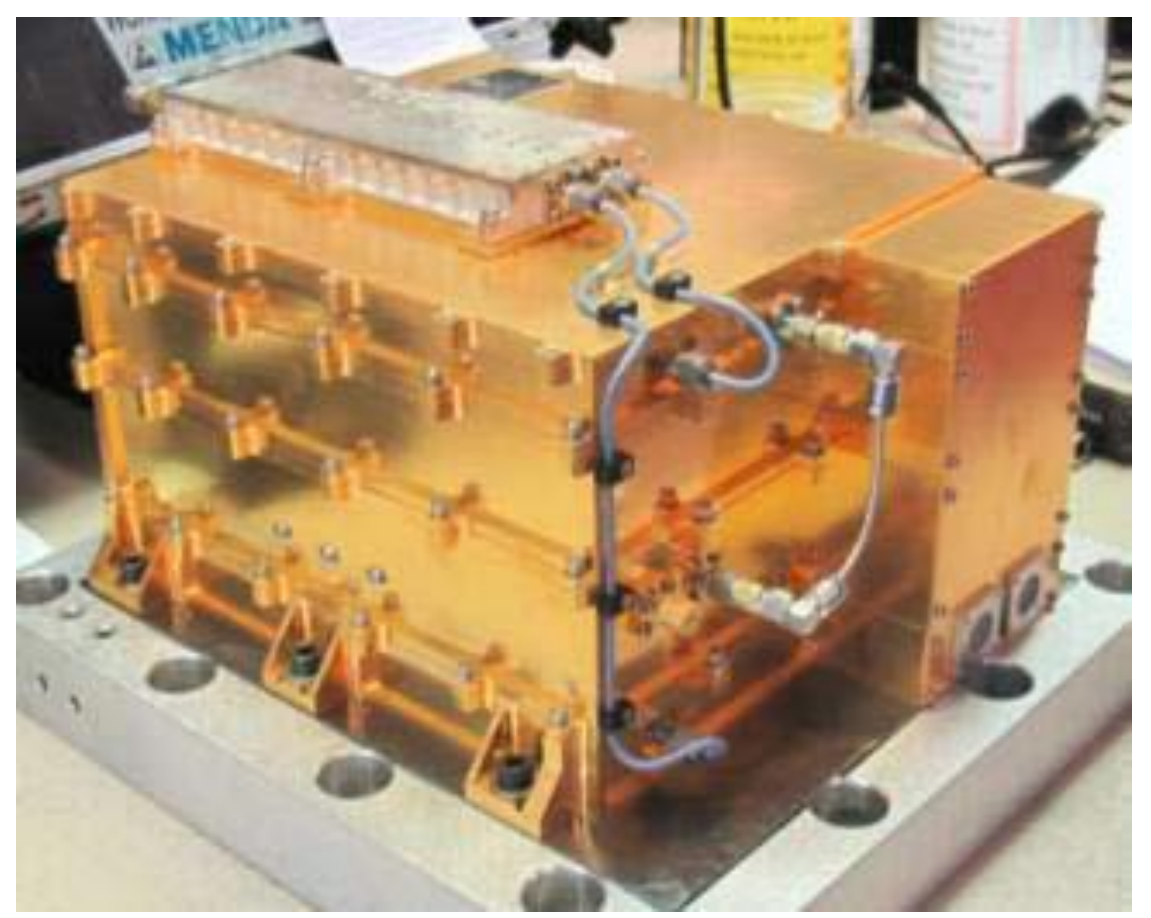

Picture Source: Reinhart, R., Wireless Innovation Forum

Technical Conference, 2013 


\section{Interacting Multiple Model (IMM) Background}

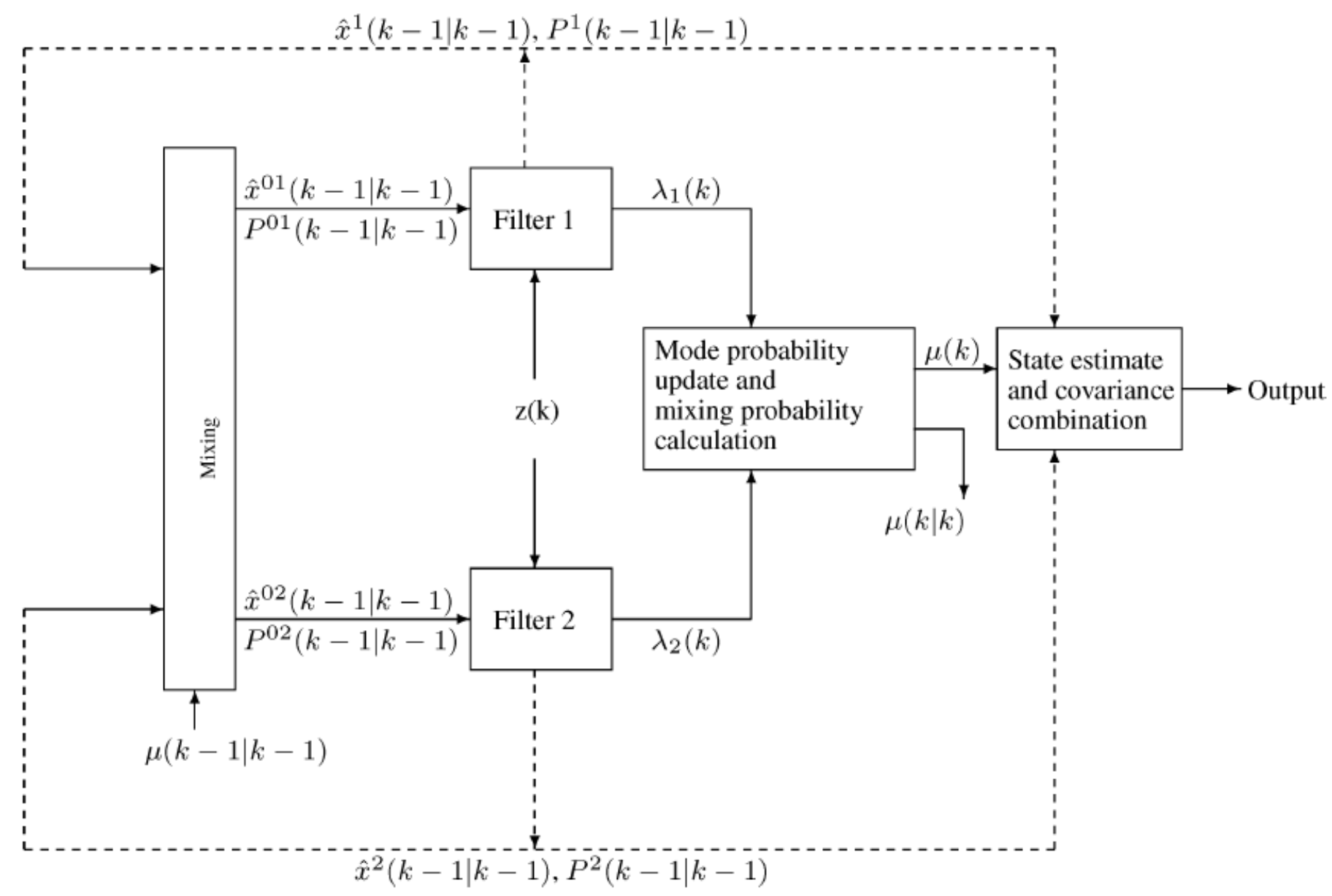

Picture Source: Sathyan, T. and Kirubarajan, T., IEEE Transactions on Circuits and Systems, 2006 


\section{Interacting Multiple Model (IMM) Background}

\begin{tabular}{|c|c|c|c|}
\hline $\begin{array}{l}\text { Step } \\
\text { Number }\end{array}$ & $\begin{array}{l}\text { Step } \\
\text { Type }\end{array}$ & $\begin{array}{l}\text { Iterations } \\
\text { Needed }\end{array}$ & Expression Executed \\
\hline MIX01 & SF & 1 & $\underline{\hat{\hat{\theta}}}(k+1 \mid k)=\underline{\mu}^{T}(k) \times \pi^{T}$ \\
\hline MIX02 & $\mathrm{SF}$ & 1 & $\overline{\pi^{T}} \times \operatorname{diag}(\underline{\mu}(k))$ \\
\hline MIX03 & $\mathrm{SF}$ & 1 & $\underline{\mu}(k)=\left(\pi^{\bar{T}} \times \operatorname{diag}(\underline{\mu})(k)\right)^{T} \times(\operatorname{diag}(\underline{\hat{\mu}}(k+1 \mid k)))^{-1}$ \\
\hline MIX04 & $\mathrm{SF}$ & 1 & $\overline{\hat{X}}_{0}(k \mid k)=\hat{X}(k \mid k) \times \mu(k)$ \\
\hline MIX05 & DF & $R^{2}$ & $\hat{X}_{j 0}(k \mid k)-\hat{X}_{i}(k \mid k)$ \\
\hline MIX06 & DF & $R^{2}$ & $\left(\hat{X}_{j 0}(k \mid k)-\hat{X}_{i}(k \mid k)\right) \times\left(\hat{X}_{j 0}(k \mid k)-\hat{X}_{i}(k \mid k)\right)^{T}+P_{i}(k \mid k)$ \\
\hline MIX07 & DF & $R^{2}$ & $\begin{aligned} P_{j 0}(k \mid k)= & {\left[\left(\hat{X}_{j 0}(k \mid k)-\hat{X}_{i}(k \mid k)\right) \times\left(\hat{X}_{j 0}(k \mid k)-\hat{X}_{i}(k \mid k)\right)^{T}+P_{i}\right] } \\
& \times \operatorname{diag}\left(\mu_{i j}\right)(k)+G\end{aligned}$ \\
\hline KF01 & DF & $R$ & $\hat{X}_{j}(k+1 \mid k)=F \times \hat{X}_{j 0}(k \mid k)$ \\
\hline KF02 & DF & $R$ & $F \times P_{j 0}(k \mid k)$ \\
\hline KF03 & DF & $R$ & $P_{j}(k+1 \mid k)=F \times P_{j 0}(k \mid k) \times F^{T}+Q$ \\
\hline KF04 & DF & $R$ & $P_{j}(k+1 \mid k) \times H_{j}^{T}$ \\
\hline KF05 & DF & $R$ & $S_{j}(k+1)=H \times P_{j}(k+1 \mid k) \times H^{T}+R_{j}$ \\
\hline KF06 & DF & $R$ & $K_{j}(k+1)=P_{j}(k+1 \mid k) \times H^{T} \times\left(S_{j}(k+1)\right)^{-1}$ \\
\hline KF07 & DF & $R$ & $P_{j}(k+1 \mid k+1)=P_{j}(k+1 \mid k)-K_{j}(k+1) \times\left(P_{j}(k+1 \mid k) \times H_{j}^{T}\right)^{T}$ \\
\hline KF08 & DF & $R$ & $e_{j}(k+1)=\underline{z}(k+1)-H \times \hat{X}_{j}(k+1 \mid k)$ \\
\hline KF09 & DF & $R$ & $\hat{X}_{j}(k+1 \mid k+1)=\hat{X}_{j}(k+1 \mid k)+K_{j}(k+1) \times \underline{e}_{j}(k+1)$ \\
\hline COM01 & DF & $R$ & $\underline{e}_{j}^{T}(k+1) \times S_{j}^{-1}(k+1) \times \underline{e}_{j}(k+1)$ \\
\hline COM02 & $\mathrm{SC}$ & $R$ & $-\frac{1}{2} e_{j}^{T}(k+1) \times S_{j}^{-1}(k+1) \times e_{j}(k+1)$ \\
\hline COM03 & $\mathrm{SC}$ & $R$ & $\exp \left(-\frac{1}{2} e_{j}^{T}(k+1) \times S_{j}^{-1}(k+1) \times e_{j}(k+1)\right)$ \\
\hline COM04 & $\mathrm{SC}$ & $R$ & $\left|S_{j}(k+1)\right|$ \\
\hline COM05 & $\mathrm{SC}$ & $R$ & $\left(\left|S_{j}(k+1)\right|\right)^{-\frac{1}{2}}$ \\
\hline COM06 & $\mathrm{SC}$ & $R$ & $\begin{aligned} \underline{L}_{j}(k+1)= & (2 \pi)^{\frac{Z}{2}}\left(\left|S_{j}(k+1)\right|\right)^{-\frac{1}{2}} \exp \left(-\frac{1}{2} e_{j}^{T}(k+1) \times S_{j}^{-1}(k+1)\right. \\
& \left.\times e_{j}(k+1)\right)\end{aligned}$ \\
\hline COM07 & $\mathrm{SF}$ & 1 & $\operatorname{diag}(\underline{L}(k+1)) \times \underline{\hat{\mu}}^{T}(k+1 \mid k)$ \\
\hline COM08 & $\mathrm{SF}$ & 1 & $\underline{\hat{\mu}}(k+1 \mid k) \times \underline{L}^{T}(\bar{k}+1)$ \\
\hline COM09 & $\mathrm{SF}$ & 1 & $\begin{aligned} & \underline{\bar{\mu}}(k+1)=\left(\operatorname{diag}\left(\hat{\underline{\mu}}(k+1 \mid k) \times \underline{L}^{T}(k+1)\right)\right)^{-1} \times(\operatorname{diag}(\underline{L}(k+1)) \\
&\left.\times \hat{\mu}^{T}(k+1 \mid k)\right)\end{aligned}$ \\
\hline COM10 & SF & 1 & $\underline{\hat{X}}(k+1 \mid k+1)=\hat{X}(k+1 \mid k+1) \times \mu(k+1)$ \\
\hline
\end{tabular}




\section{Implementation Trade Studies}

$$
A=\left[\begin{array}{ll}
a_{11} & a_{12} \\
a_{12} & a_{22}
\end{array}\right] \quad A^{-1}=?
$$

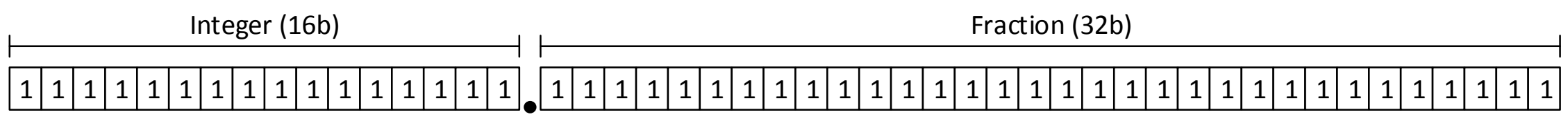
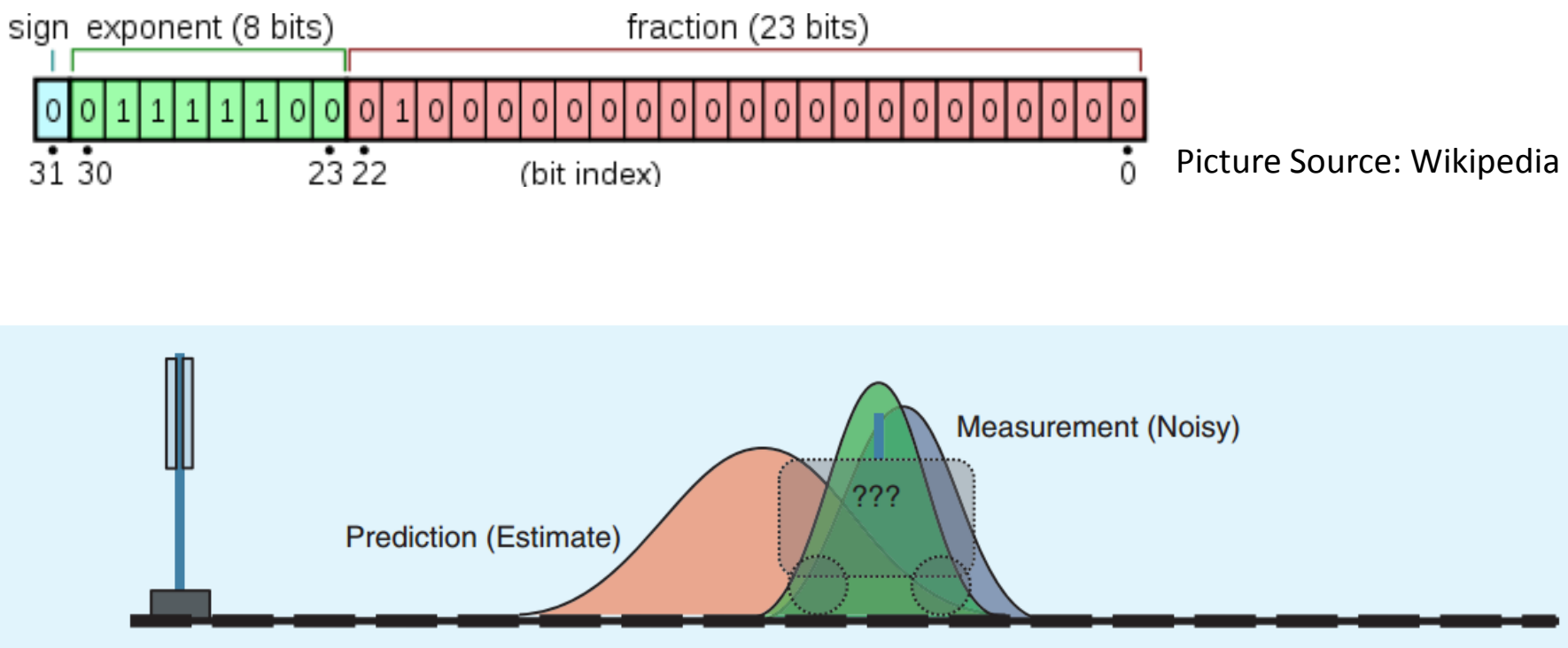

Picture Source: Faragher, R., IEEE Signal Processing Magazine, 2012 


\section{Schur Complement Architecture}
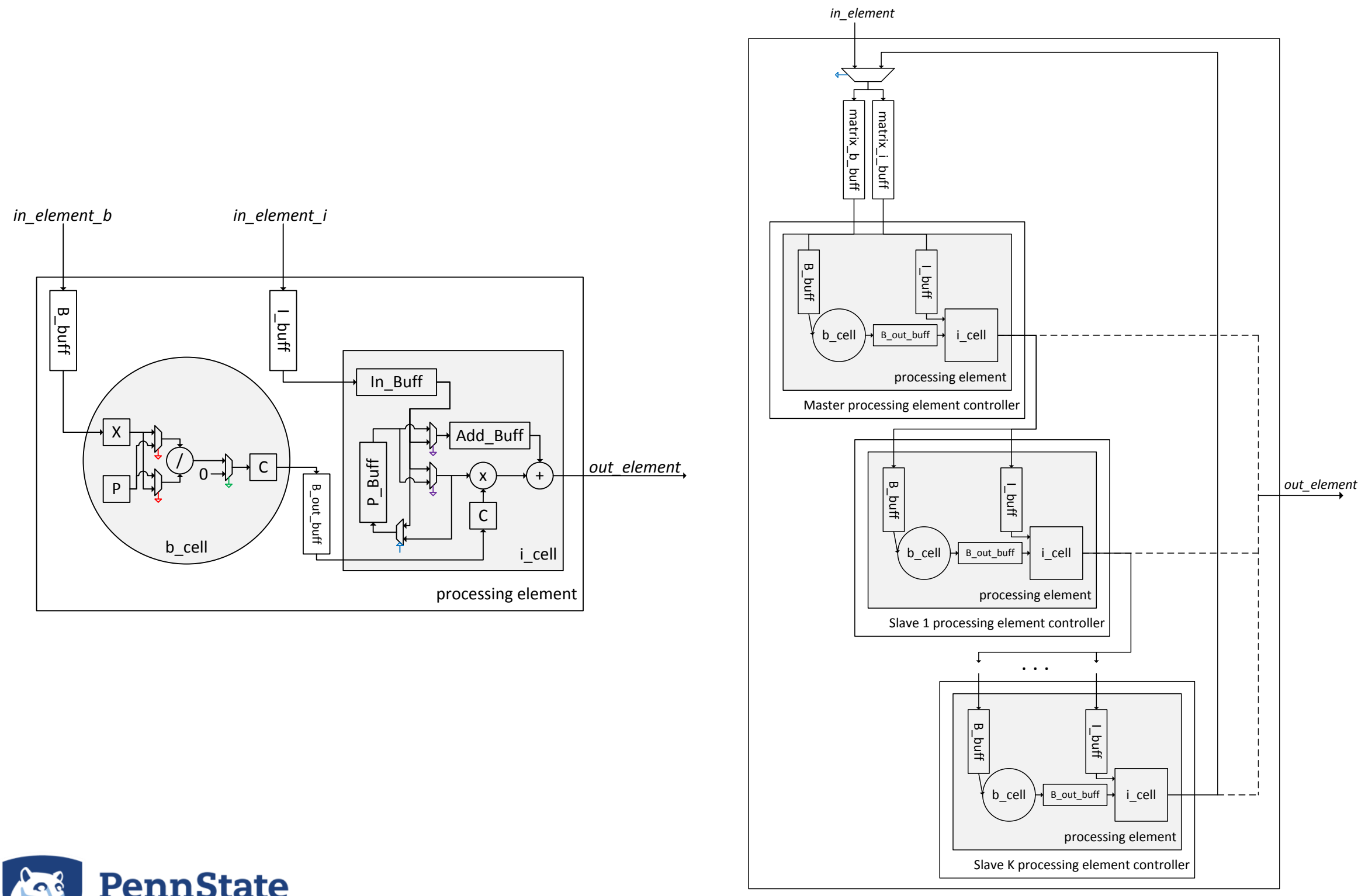


\section{Schur Complement Analysis}

\begin{tabular}{cccc|ccc|c}
\hline $\begin{array}{c}\text { Multiplier } \\
\text { Latency }\end{array}$ & $\begin{array}{c}\text { Addition } \\
\text { Latency }\end{array}$ & $\begin{array}{c}\text { Divider Cycles } \\
\text { Per Operation }\end{array}$ & $\begin{array}{c}\text { Divider } \\
\text { Latency }\end{array}$ & $\begin{array}{c}\text { Occupied } \\
\text { Slices }\end{array}$ & $\begin{array}{c}\text { Slice Flip } \\
\text { Flops }\end{array}$ & $\begin{array}{c}\text { 4-Input } \\
\text { LUTs }\end{array}$ & $\begin{array}{c}\text { Max Clock } \\
\text { Frequency (MHz) }\end{array}$ \\
\hline 1 & 1 & 14 & 14 & 754 & 541 & 1299 & 50.742 \\
3 & 5 & 14 & 14 & 829 & 925 & 1340 & 133.233 \\
3 & 7 & 26 & 27 & 849 & 1090 & 1328 & 152.879 \\
6 & 7 & 26 & 27 & 910 & 1201 & 1343 & 175.389 \\
\hline
\end{tabular}




\section{IMM Filter Architecture}

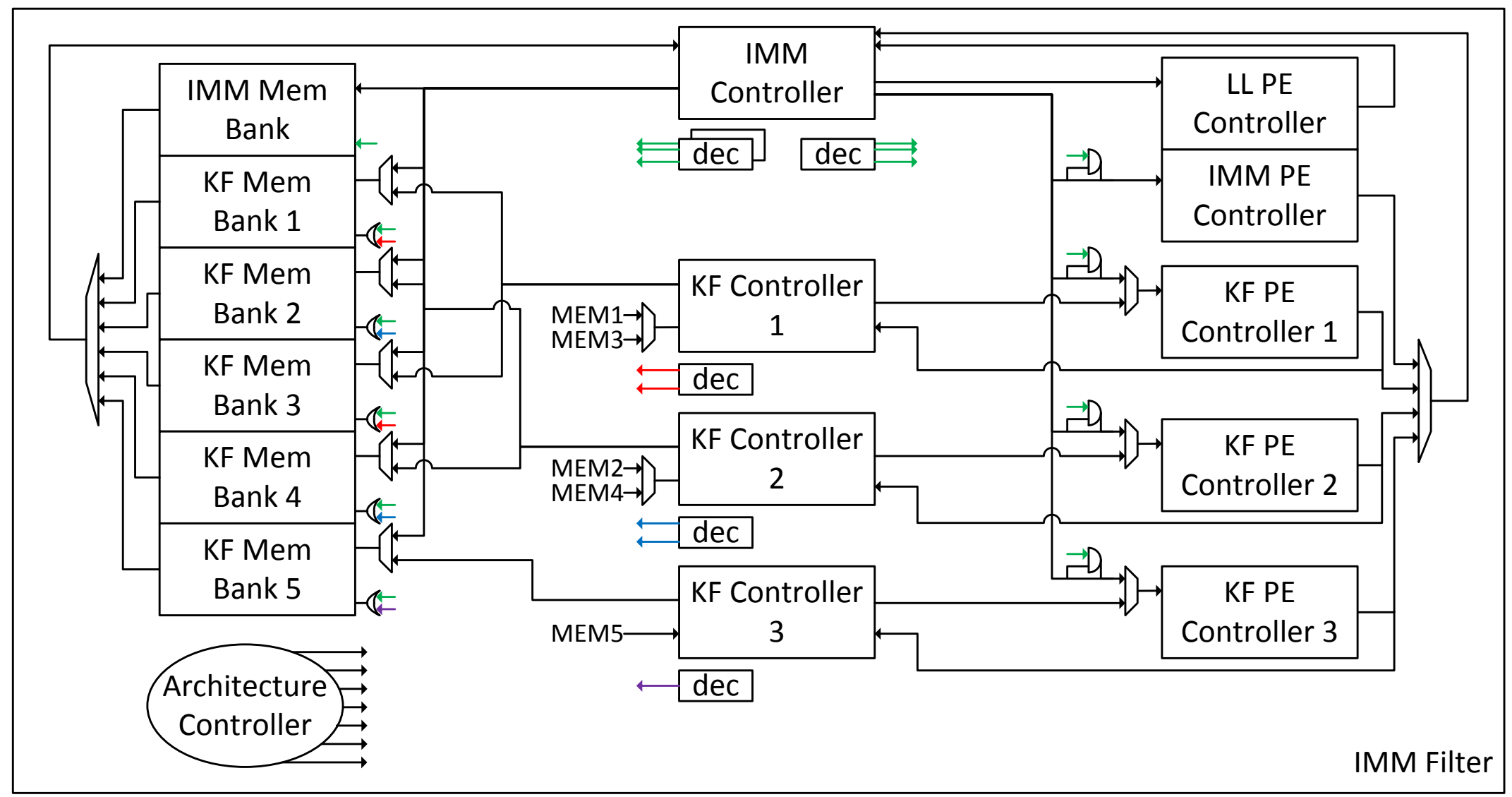




\section{IMM Analysis}
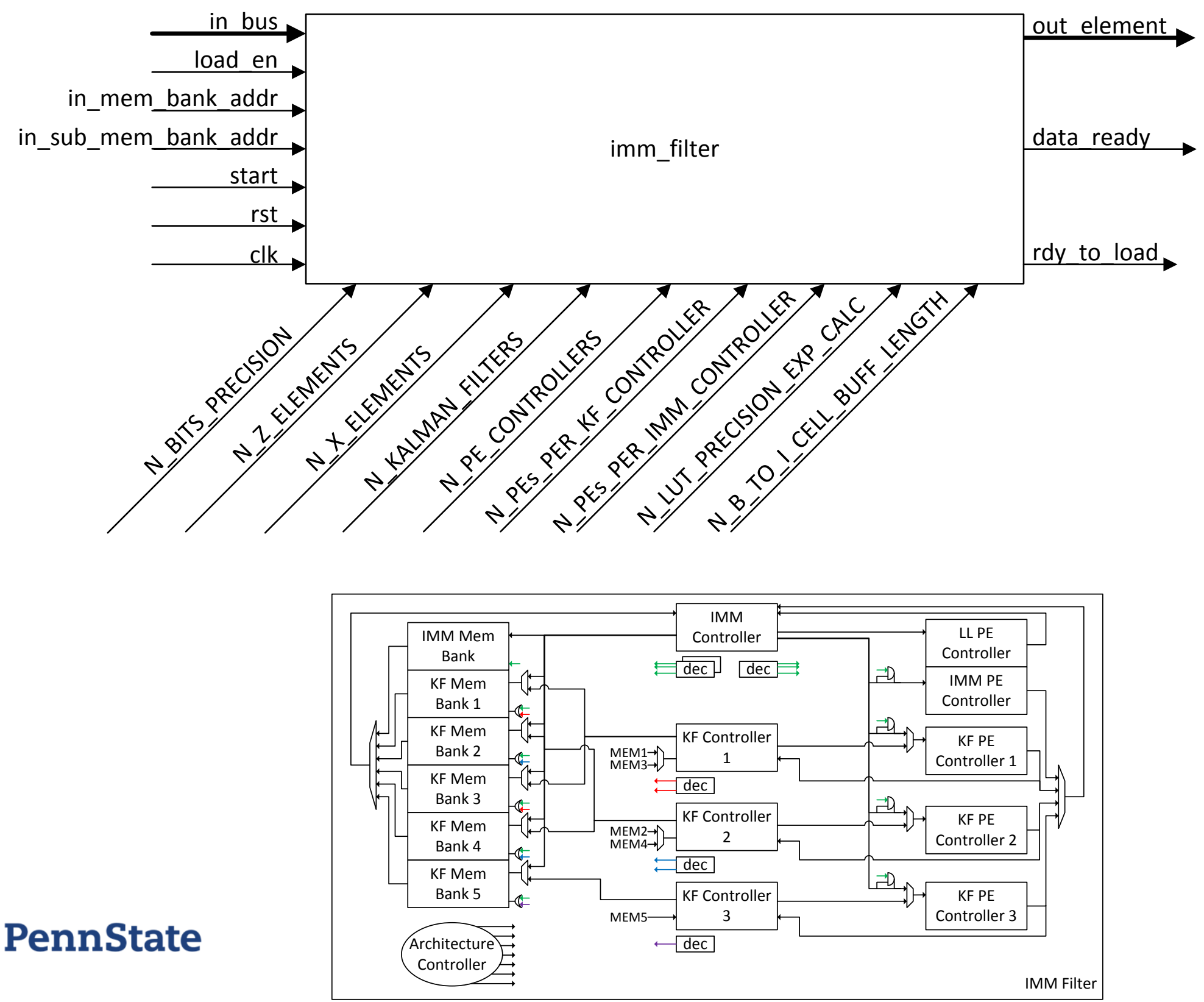


\section{Example Scenario}

State Model

Constant Acceleration Model

Available Measurements SNR, SIR

System Models

Clear Sky, Rain Fade, Ionospheric Scintillation, Tropospheric Scintillation, Multipath Fading

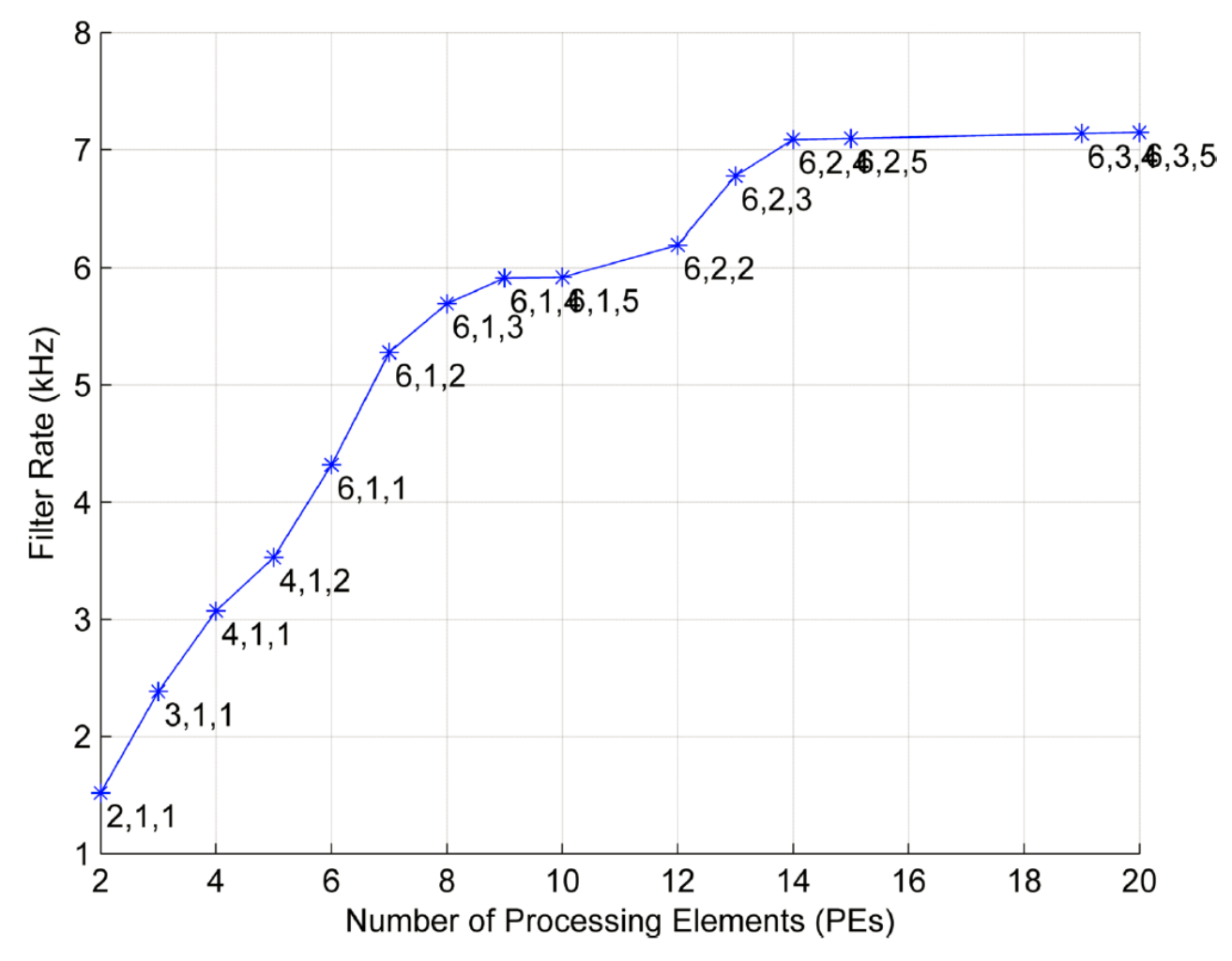




\section{IMM Analysis}

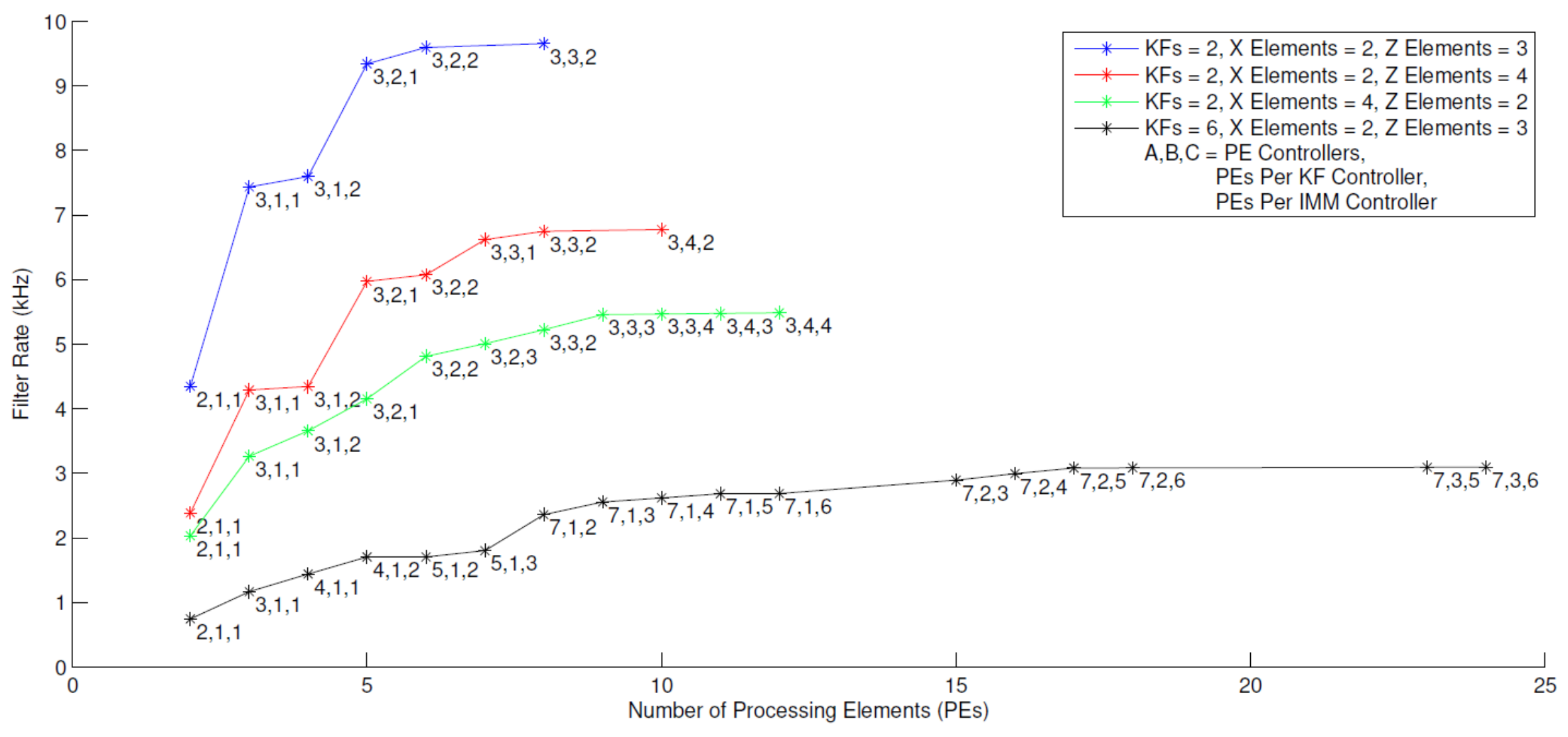




\section{Summary}

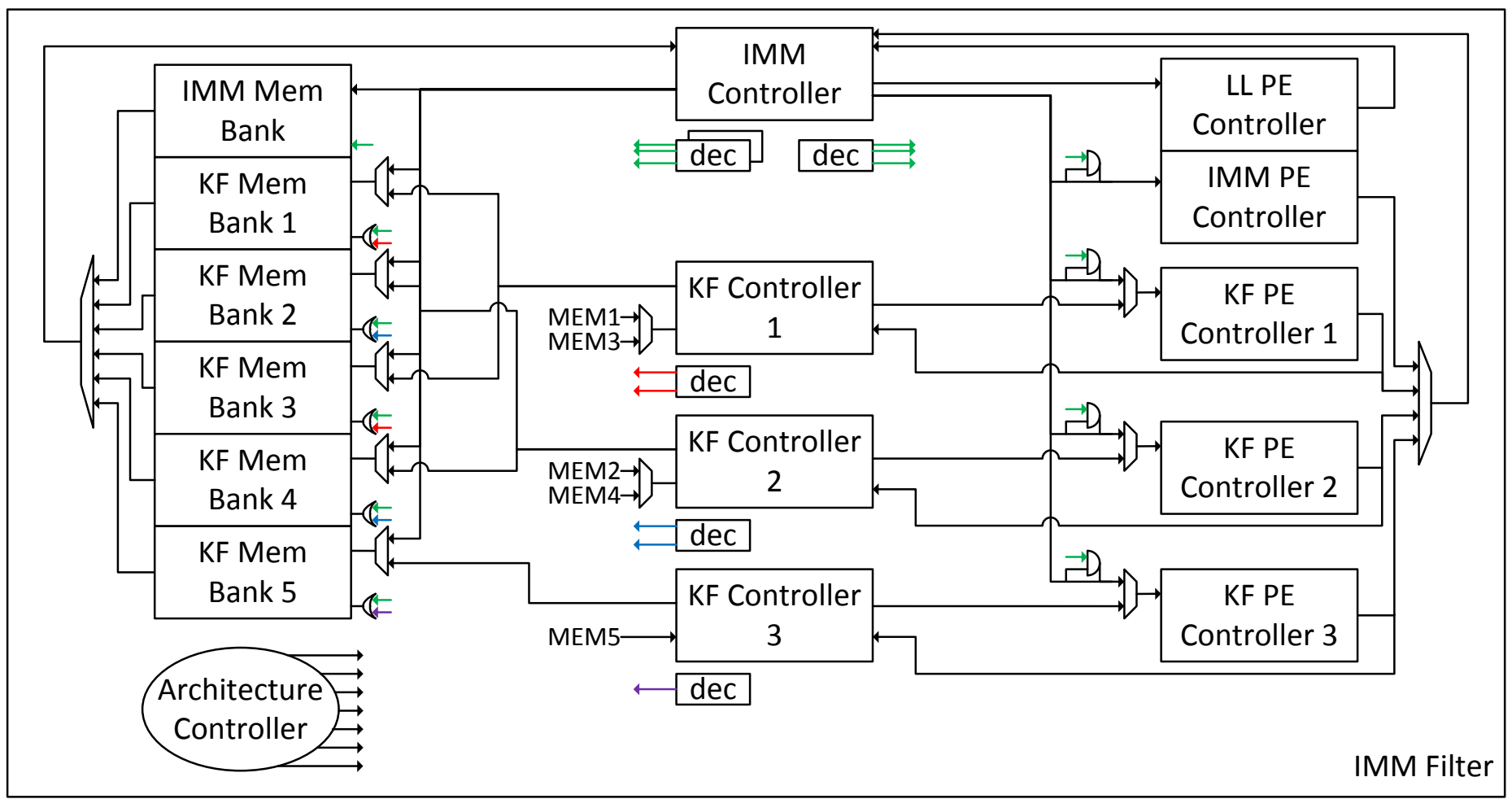

If you're interested, contact me at:

Tim Hackett: tmh5344@psu.edu 